\title{
A NOTE ON AN EXAMPLE OF STALLINGS ${ }^{1}$
}

\author{
R. B. SHER
}

1. Introduction. R. H. Bing [1] has shown that Euclidean 3-space $E^{3}$ does not contain an uncountable collection of pairwise disjoint wild closed surfaces. J. Stallings [3] gave an example of an uncountable collection of pairwise disjoint wild disks in $E^{3}$. J. Martin [2] showed that in an uncountable collection of pairwise disjoint disks all but a countable number of elements of the collection lie on a 2sphere. In a review of Martin's paper, O. G. Harrold pointed out that what is apparently unknown is whether an uncountable collection of pairwise disjoint disks must contain a pair of equivalently imbedded elements. The purpose of this note is to show that it need not.

We use $E^{3}$ to denote the set of all 3-tuples $\left(x_{1}, x_{2}, x_{3}\right)$ of real numbers with the usual topology. We identify $E^{1}$ (the reals) with the $x_{1}$-axis in $E^{3}$, and $E^{2}$ (the plane) with the $x_{1} x_{2}$-coordinate plane. We use coordinates $\left(x_{1}, x_{2}\right)$ rather than $\left(x_{1}, x_{2}, 0\right)$ to represent points of $E^{2}$.

Compact sets $H$ and $K$ are equivalently imbedded in $E^{3}$ if there is a homeomorphism of $E^{3}$ on to itself which carries $H$ onto $K$. If there is a polyhedron $P$ so that $H$ and $P$ are equivalently imbedded, we say that $H$ is tame. Otherwise, $H$ is wild. The set $H$ is locally tame at $p \in H$ if there is a neighborhood $N$ of $p$ so that the closure of $N \cap H$ is tame. If $W$ is a closed subset of $H$ and $H$ is locally tame at each point of $H-W, H$ is said to be locally tame modulo $W$.

If $D$ is a disk or 3-cell, Bd $D$ will denote the boundary simple closed curve or 2-sphere of $D$. We use $\mathrm{Cl} H$ for the closure of the point set $H$.

2. Construction. Let $M$ be the usual "middle-third" Cantor set in $[0,1] \subset E^{1}$. That is, $M=\cup_{i=0}^{\infty} K_{i}$ where $K_{0}=[0,1]$ and $K_{i}$ is the union of the $2^{i}$ pairwise disjoint closed intervals obtained by removing the middle-third open interval from each component of $K_{i-1}$ if $i=1,2,3, \cdots$. If $i=1,2,3, \cdots$, we shall denote the $2^{i}$ components of $K_{i}$ by $L_{1}^{i}, L_{2}^{i}, L_{3}^{i}, \cdots, L_{2}^{i} i$ where each point of $L_{j}^{i}$ precedes each point of $L_{j+1}^{i}$ if $j=1,2, \cdots$ or $2^{i}-1$. We denote $L_{j}^{i} \cap M$ by $M_{j}^{i}$.

-We now define a sequence $N_{1}, N_{2}, N_{3}, \cdots$ of Cantor sets in $E^{2}$ so that

$$
N=\mathrm{Cl}\left(\bigcup_{i=1}^{\infty} N_{i}\right)=M \cup\left(\bigcup_{i=1}^{\infty} N_{i}\right)
$$

Received by the editors February 14, 1967.

1 This research was supported by NSF Grant GP-6016. 
is a Cantor set. The set $N_{i}$ is defined to be

$$
\left[\bigcup_{k=1}^{2^{i-1}}\left(M_{2 k-1}^{i} \times\left\{2 / 3^{i}\right\}\right)\right] \cup\left[\bigcup_{k=1}^{2^{i-1}}\left(M_{2 k}^{i} \times M_{2}^{i}\right)\right] .
$$

Now let $\left\{S_{\alpha}\right\}$ be the set of all infinite sequences each of whose terms is either 0 or 1 . If $S_{\alpha}$ is the sequence $m_{1}, m_{2}, m_{3}, \cdots$, we associate the sequence $L_{\bar{m}_{1}}^{1} \supset L_{\bar{m}_{2}}^{2} \supset L_{\bar{m}_{2}}^{3} \supset \ldots$ with $S_{\alpha}$ where $\bar{m}_{j}$ is odd if $m_{j}$ is 0 and even if $m_{j}$ is 1 . The correspondence $S_{\alpha} \rightarrow \bigcap_{i=1}^{\infty} L_{\bar{m}_{i}}^{i}=p_{\alpha} \in M$ is a 1-1 correspondence between the elements of $\left\{S_{\alpha}\right\}$ and the points of $M$. Notice that the subset $\left\{S_{\alpha}^{\prime}\right\}$ of $\left\{S_{\alpha}\right\}$, consisting of those members of $\left\{S_{\alpha}\right\}$ in which no 1's appear adjacent to one another, has cardinality of the continuum. ${ }^{2}$

Now let $Q$ be a 3-cell in $E^{3}$ such that Bd $Q$ is locally tame modulo a Cantor set $C$ and so that any arc on $\mathrm{Bd} Q$ which intersects $C$ is wild. An example of such a cell is described by Stallings in [3]. There is a homeomorphism $h: Q \rightarrow Q^{\prime}=\left\{\left(x_{1}, x_{2}, x_{3}\right) \mid 0 \leqq x_{i} \leqq 1, i=1,2,3\right\}$ so that $h(C)=N$. Let $D_{\alpha}^{\prime}$ be the intersection of $Q^{\prime}$ with the plane $x_{1}=p_{\alpha}^{\prime}$ where $p_{\alpha}^{\prime}$ is associated with the sequence $S_{\alpha}^{\prime}$, and let $D_{\alpha}=h^{-1}\left(D_{\alpha}^{\prime}\right)$.

The set $\left\{D_{\alpha}\right\}$ is an uncountable collection of pairwise disjoint wild disks in $E^{3}$. The boundary of each $D_{\alpha}$ is locally tame modulo $D_{\alpha} \cap C$. A description of the wild set of $\mathrm{Bd} D_{\alpha}$ is given by examining the sequence $S_{\alpha}^{\prime}$. To each 0 in $S_{\alpha}^{\prime}$ there corresponds an isolated wild point of Bd $D_{\alpha}$, and to each 1 in $S_{\alpha}^{\prime}$ there corresponds a Cantor set of wild points, and $S_{\alpha}^{\prime}$ describes the relative order of these "components" of the wild set on $\mathrm{Bd} D_{\alpha}$. The fact that a space homeomorphism taking one disk onto another must necessarily carry wild points to wild points and the choice of the sequences $\left\{S_{\alpha}^{\prime}\right\}$ shows that no pair of the disks $\left\{D_{\alpha}\right\}$ are equivalently imbedded in $E^{3}$.

\section{REFERENCES}

1. R. H. Bing, $E^{3}$ does not contain uncountably many mutually exclusive wild surfaces, Abstract 801t, Bull. Amer. Math. Soc. 63 (1957), 404.

2. J. Martin, A note on uncountably many disks, Pacific J. Math. 13 (1963), 13311333. 186.

3. J. R. Stallings, Uncountably many wild disks, Ann. of Math. (2) 71 (1960), 185-

\section{UNIVERSITY OF GEORGIA}

2 In fact, as pointed out by the referee, the image of the set $\left\{S_{\alpha}^{\prime}\right\}$ under the 1-1 correspondence $S_{\alpha} \rightarrow p_{\alpha}$ is a Cantor set thus showing that there is an uncountable compact collection of pairwise disjoint disks, no two of which are equivalently imbedded. 